\title{
Study of ANN Configuration on Performance of Smart MIMO Channel Estimation for Downlink LTE-Advanced System
}

\author{
Nirmalkumar S. Reshamwala ${ }^{1}$, Pooja S. Suratia ${ }^{2}$, Satish K. Shah ${ }^{3}$ \\ Department of Electrical Engineering ${ }^{1,2,3}$ \\ The Maharaja Sayajirao University of Baroda Vadodara, Gujarat, India. \\ reshamwala.nirmal01@gmail.com¹, poojasuratia@yahoo.com², satishkshah_2005@yahoo.com³
}

\begin{abstract}
Long-Term Evolution (LTE) is the next generation of current mobile telecommunication networks. LTE has a new flat radio-network architecture and significant increase in spectrum efficiency. In this paper, performance analysis of robust channel estimators for Downlink Long Term EvolutionAdvanced (DL LTE-A) system using three Artificial Neural Network ANN Architectures: Feed-forward neural network, Cascade-forward neural network and Layered Recurrent Neural Network (LRN) are adopted to train the constructed ANNs models separately using Back-Propagation Algorithm. The methods use the information got by the received reference symbols to estimate the total frequency response of the channel in two important phases. In the first phase, the proposed ANN based method learns to adapt to the channel variations, and in the second phase it estimates the channel matrix to improve performance of LTE. The performance of the estimation methods is evaluated by simulations in Vienna LTE-A DL Link Level Simulator. Performance of the proposed channel estimator, Layered Recurrent Neural Network is compared with traditional Least Square (LS) algorithm and ANN based other estimator like Feed-forward neural network and Cascade-forward neural network for Closed Loop Spatial Multiplexing-Single User Multi-input Multioutput $(2 \times 2$ and $4 \times 4)($ CLSM-SUMIMO) in terms of throughput. Simulation result shows LRN gives better performance than other ANN based estimations methods and LS.
\end{abstract}

Index Terms - LTE-A, MIMO, Artificial Neural Network (ANN), Back-Propagation, Layered Recurrent Neural Network (LRN), Feed-forward neural network, Cascade-forward neural network

\section{INTRODUCTION}

The Long Term Evolution (LTE) is a step towards the fourth generation (4G) of mobile radio technologies to increase the spectral efficiency, user capacity, low latency and to obtain higher throughput. LTE-Advanced (LTE-A) refers to the evolved version of LTE that is being developed by 3rd Generation Partnership Project (3GPP), a new standard as the evolution of the current network architecture of mobile communications, GSM/HSPA. LTE Release10 [1], [2] is set to provide higher bit rates in a cost efficient way and, at the same time, completely fulfill the requirements set by International Telecommunication Union (ITU) for International Mobile Telecommunications-Advanced (IMT Advanced) also referred to as 4G [2], [3]. The features supported by LTE-A are given in [3]. Release 10 support enhanced MIMO Techniques with 8 X8 in downlink and upto 4X4 in uplink with wider bandwidths, enabled by carrier aggregation. It achieves maximum Peak data rate 1 Gbps for downlink and 500 Mbps for uplink. LTE-Advanced uses an Orthogonal Frequency Division Multiplex Access (OFDMA) radio interface in downlink and the Single-Carrier Frequency Division Multiple Access (SC-FDMA) for the uplink [4].

The receiver in MIMO system requires the knowledge of Channel State Information (CSI) in order to recover the original transmitted signal properly without noise. In certain channel estimation methods, pilot symbols are inserted and transmitted over the channel, and are estimated at the receiver in order to recover original transmitted symbols [4], [5]. The most traditional efficient training based methods are the Least Squares (LS) method [6], [7], Minimum Mean Square Error (MMSE) method [8], [9] and Adaptive Filtering channel estimation method [10], [11]. In this paper, Channel estimation by different Artificial Neural Networks configuration has been deployed in LTEAdvance system, with different neural network architectures [12], [13], [14], [15], [16]. In this contribution, we propose Smart MIMO Channel Estimation using ANN Configuration for Downlink LTE-Advanced System. The principle of this method is to exploit the information provided by the received reference symbols to estimate the channel response using result of conventional LS Estimator.

In section II, the LTE-A Downlink Physical Layer and The Vienna LTE-A Link Level Simulator is explained. Section III describes different channel estimation techniques like Least Square (LS) and ANN 
based techniques. Followed by simulation results and performance analysis of proposed ANN based channel estimation techniques in Section IV. Finally Conclusion and further development is discussed in Section V.

\section{LTE-A DOWNLINK PHYSICAL LAYER AND VIENNA LTE-A LINK LEVEL SIMULATOR}

The LTE Physical Layer is highly efficient means of conveying both data and control information between an enhanced base station (called eNodeB in LTE terminology) and mobile user equipment (UE) [17], [18]. Although, the LTE physical layer specification describes both FDD (Frequency Division Duplex) and TDD (Time Division Duplex), the study in this paper is focused on FDD, thus only LTE physical layer with FDD is discussed below. Furthermore, only downlink data transmission is considered. Downlink LTEAdvance system is based on OFDMA air interface transmission scheme [3]. OFDMA-MIMO system is described in [5]. The orthogonal frequency subcarriers are used to share spectrum among users using access technique. The LTE-A Physical layer employs advanced technologies of wireless cellular systems [19], [20].

LTE-A Downlink Physical Layer [19], [20] is described as shown in Fig. 1. Physical channel processing at eNodeB consists of Scrambling which breaks long strings of $1 \mathrm{~s}$ and 0 s into scrambled bits, Modulation converts scrambled bits into complexvalued symbols uses either QPSK, 16-QAM or 64QAM modulation, Layer mapper and precoder performs symbol transformations to enable MIMO transmission techniques, Resource element (RE) mapper maps the symbols to the appropriate locations in the timefrequency Resource grid, OFDM signal mapper generates time domain baseband signals for each antenna port (Antenna Port 0, 1, 2 or 4) for transmission [5]. Similarly at UE descrambling, demodulation and demapper operations and MIMO Receiver processing to receive the transmitted data streams. LTE-A Downlink modulation is based on OFDMA which provides multi user access, robustness to time dispersion of radio channel, and low complexity for receiver design. Also, the multicarrier concept enables the operation of LTE in various system bandwidths up to $20 \mathrm{MHz}$ by adapting the number of subcarriers used. In Closed Loop Spatial Multiplexing-CLSM, The uplink feedback values Channel Quality Indicator (CQI), Rank Indicator (RI) and Precoding Matrix Indicator (PMI) are calculated at the receiver, and are feedback to the eNodeB. In CLSM, Each transmit antenna transmits a different data stream. This technique significantly increases the peak data rate over the radio link. (For instance, 2x2 MIMO effectively increases the peak data rate by a factor of two). It requires high signal-to-noise-plus-interference ratio (SNIR) radio conditions in order to be effective. Although the data streams are transmitted simultaneously at the same frequency, the receiver can nevertheless detect the different streams received via different antennas- hence the name spatial multiplexing [20].

LTE-A Downlink Physical channels convey information from higher layers in the LTE stack. Each physical channel defines algorithms for bit scrambling, modulation, layer mapping, CDD (Cyclic Delay Diversity), precoding and resource element assignment. There are three downlink physical channels in LTE [5], [21].

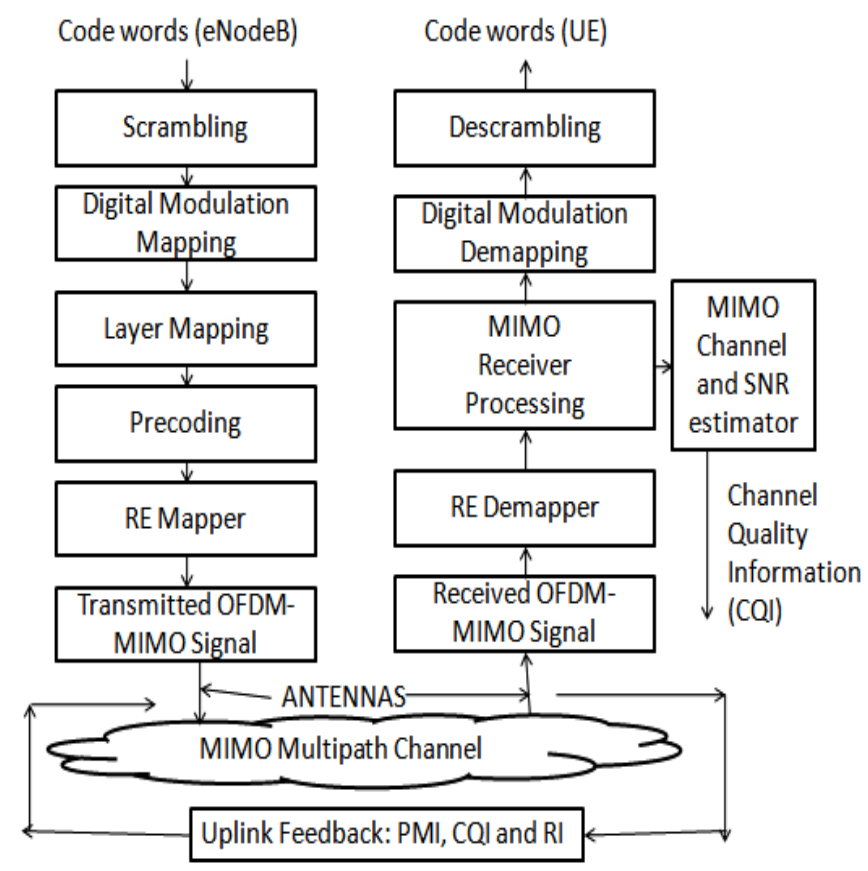

Figure 1. LTE-A Downlink Physical Layer [18-19]

Link Level Simulator [22] is used to emulate the transmission of user data and control information from an eNodeB transmitter to a UE receiver modeling the physical layer with high precision [16], [23]. The Link level parameters for the presented simulations are as shown in Table I. Transmission modes in the first release of LTE according to [17], [24] are configured in this LTE-A Downlink Link Level Simulator. Simulated MIMO scheme followed CLSM (Close-Loop Spatial Multiplexing) transmission mode specified by the 3GPP [17], [24], [25].

\section{CHANNEL ESTIMATION TECHNIQUE BASED ON LS ESTIMATOR AND ARTIFICIAL NEURAL NETWORKS}

\section{A. Least Square Channel Estimation}

Least square channel estimator is obtained by minimizing the square distance between the received signal $\bar{Y}$ and the transmitted signal $\underline{X}$ as follows [6], [25]

$$
\begin{aligned}
& \min _{H^{T}} J(H)=\min _{H^{T}}\left\{|\bar{Y}-\underline{X} \bar{H}|^{2}\right\} \\
& =\min _{H^{T}}\left\{(\bar{Y}-\underline{X} \bar{H})^{T}(\bar{Y}-\underline{X} \bar{H})\right\}
\end{aligned}
$$

where, $(.)^{T}$ is the conjugate transpose operator. 
By differentiating expression (1) with respect to $\bar{H}^{T}$ and finding the minima, we obtain

$$
\frac{\partial}{\partial \bar{H}^{T}} J(H)=-\underline{X}^{T} \bar{Y}+\underline{X}^{T} \underline{X} \bar{H}=0
$$

Finally, the LS channel estimation is given by [5], [24]

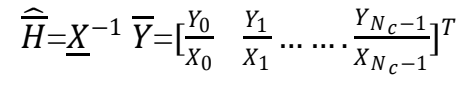

In general, LS channel estimation technique for OFDM systems has low complexity but it suffers from a high mean square error (MSE) [6].

\section{B. Artificial Neural Network}

An artificial neuron is defined as follows: It receives a number of inputs either from original data, or from the output of other neurons in the neural network. Each input comes via a connection that has strength (weight); these weights correspond to synaptic efficacy in a biological neuron. Each neuron also has a single threshold value. The weighted sum of the inputs is formed, and the threshold subtracted, to compose the activation of the neuron (also known as the postsynaptic potential, or PSP, of the neuron) [33], [34], [35].

The activation signal is passed through an activation function (also known as a transfer function) to produce the output of the neuron. Inputs and outputs correspond to sensory and motor nerves such as those coming from the eyes and leading to the hands. There can be hidden neurons that play an internal role in the network. The input, hidden and output neurons need to be connected together [33], [34], [35].

Fig. 2 shows the basic and simple model of an Artificial Neuron present in the layers of the Artificial Neural Network.

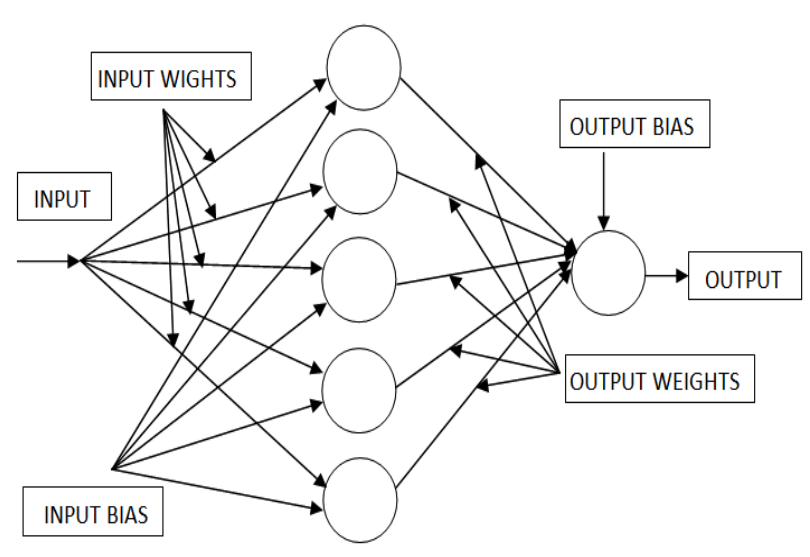

Figure 2. Simple model of an Artificial Neuron

A simple network has a feed-forward structure; signals flow from inputs, forwards through any hidden units, eventually reaching the output units. Such a structure has stable behavior. However, if the network is recurrent (contains connections back from later to earlier neurons) it can be unstable, and has very complex dynamics [33].

When the network is executed (used), the input variable values are placed in the input units, and then the hidden and output layer units are progressively executed. Each of them calculates its activation value by taking the weighted sum of the outputs of the units in the preceding layer, and subtracting the threshold. The activation value is passed through the activation function to produce the output of the neuron. When the entire network has been executed, the outputs of the output layer act as the output of the entire network [33], [34], [35].

- $\quad$ A Feed-forward neural Network (FFNN) [34], [35] is one whose topology has no closed paths and number of hidden layer as per requirement and input nodes are connected to the output nodes without any feedback paths. The Back-Propagation Algorithm (BPA) uses the steepest-descent method to reach a global minimum with energy function of MSE. The flowchart of the BPA is given in [36].

- In Cascade-forward neural networks, these is similar to feed forward networks such as BackPropagation Neural networks (BPNN) with the exception that they have a weight connection from the input and every previous layer to the following layers [37], [38], [39].

- In Layered Recurrent Neural Network (LRN), the method of training is similar to that of the FFNN model. In addition to this, a neuron taking input from the output layer and connected to the hidden layer as shown in Fig. 3. Initially, the weights assigned are small random numbers. The inputs are presented to the input units and the output from the network is calculated just as in the case of the FFNN [39], [40], [41].

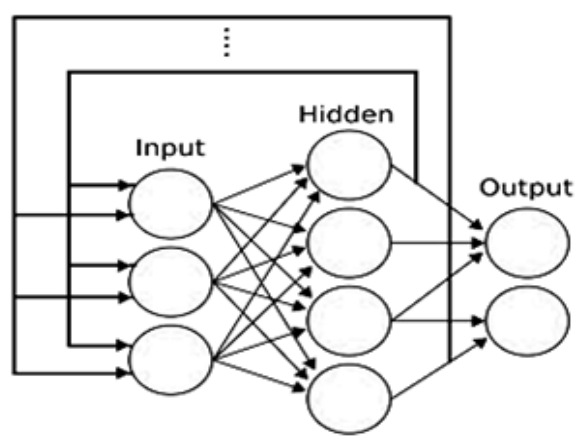

Figure 3. Layered Recurrent Neural Network (LRN)

\section{Channel Estimation using Different ANN}

The simulation program of each one of conducted Back-Propagation training algorithm (Feed-Forward Network, Cascade-Forward Network and Layered Recurrent Neural Network) includes the following steps [39]:

- First of all, estimate channel using LS estimator using transmitted signal and received signal after 
passing through AWGN and Flat Rayleigh channel and adding noise as shown in Fig. 4.

- Initialization of network weights, learning rate and Threshold error. Set iterations to zero.

- $\quad$ Take received signal MIMO matrix as an input and estimated MIMO matrix as a target

- $\quad$ Total_error = zero; iterations-> iterations+1

- $\quad$ Feed it to input layer units.

- Initialize the target output of that ANN.

- Calculate the outputs of hidden layer units.

- Calculate the outputs of output layer units.

- $\quad$ Calculate the error = desired output - actual output Total_error -> Total_error + error

- Calculate delta sigma of output neurons. Then adjust weights between output and hidden layer units.

- Calculate delta sigma of hidden layer units. Then adjust weights between hidden and input layer units.

- While there are more matrix in the file, go to step 4 .

- if Threshold error >= Total_error then stop, otherwise go to step $3^{\text {rd }}$ steps in this algorithm.

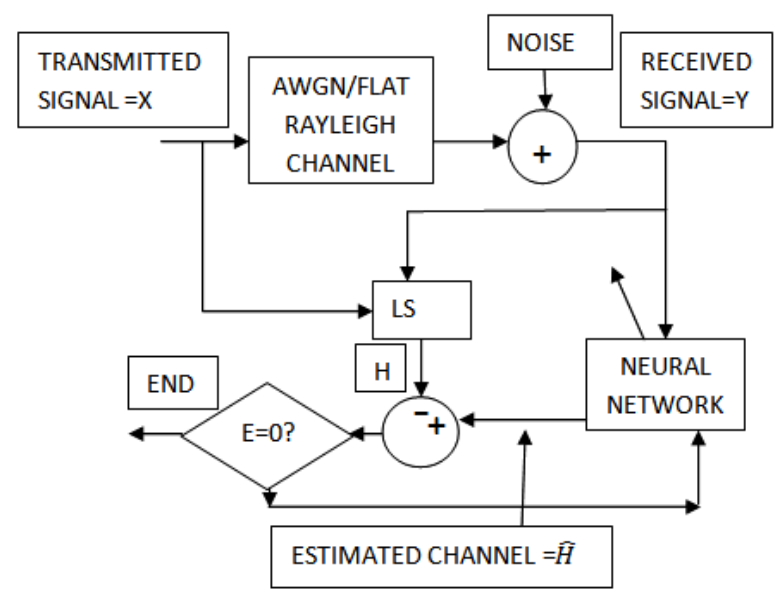

Figure 4. Channel Estimation using ANN [19, 20]

The estimator uses the information provided by received reference symbols of sub channels to estimate the total channel frequency response. The input of the neural network is the received reference symbols $\mathrm{Y}$, target of ANN is channel estimated by LS [19], [20], [42] Simulation Results

\section{A. Simulation Parameters for LS Channel Estimator}

The simulation parameters for Vienna LTE-A Link level simulator for LS channel estimator are listed in Table I.

TABLE I SiMULATION PARAMETERS FOR LS CHANNEL ESTIMATOR

\begin{tabular}{|c|c|}
\hline Parameters & Value \\
\hline Number of Sub frames & 20 \\
\hline SNR Range & {$[0,5,10,15,20,25,30]$ for } \\
& AWGN Channel \\
\cline { 2 - 2 } & {$[0,5,10,15,20,25,30,35,40]$ for } \\
& Flat Rayleigh Channel \\
\hline Transmission Bandwidth & $1.4 \mathrm{MHz}$ \\
\hline Sub-frame duration & $1 \mathrm{~ms}$ \\
\hline
\end{tabular}

\begin{tabular}{|c|c|}
\hline Sub-carrier spacing & $15 \mathrm{kHz}$ \\
\hline FFT size (N) & 128 \\
\hline $\begin{array}{c}\text { Number of occupied sub- } \\
\text { carriers }\end{array}$ & 72 \\
\hline TTI length & $1 \mathrm{~ms}$ \\
\hline Simulation Configuration & SU-MIMO \\
\hline Antenna schemes & CLSM-MIMO (2x2 and 4x4) \\
\hline MIMO receiver equalizer & ZF (Zero Forcing) \\
\hline Channel type & AWGN and Flat Rayleigh \\
\hline
\end{tabular}

\section{B. ANN based simulation parameters}

The simulation parameters for different Neural Networks are as in Table II.

TABLE II SimUlation PARAMETERS FOR FFNN, CASCADEFORWARD ANN AND LRN BASED ESTIMATORS

\begin{tabular}{|c|c|}
\hline Parameters & DIFFERENT ANN ARCHITECTURES \\
\cline { 2 - 3 } & Value \\
\hline Number of inputs & 1 \\
\hline $\begin{array}{c}\text { Number of hidden } \\
\text { layers }\end{array}$ & 1 \\
\hline $\begin{array}{c}\text { Number of neurons in } \\
\text { hidden layer }\end{array}$ & 5 \\
\hline $\begin{array}{c}\text { Epoch number / } \\
\text { Iteration }\end{array}$ & Trainscg \\
\hline $\begin{array}{c}\text { Training Function / } \\
\text { Algorithm }\end{array}$ & Mean Square Error \\
\hline Performance \\
\hline
\end{tabular}

In Feed-forward neural network, Cascade-forward neural network and Layered Recurrent Neural Network (LRN), the ANN is trained with reference symbols, which is complex type matrix. The target sample set is presented to the ANN in the form of result or estimated channel obtained by Least Square (LS) complex type matrix. The learning of the ANN is done in the training phase during which the ANN adjusts its weights according to training algorithm. The ANN is trained for 1000 epochs.

In this paper, Comparative Effect of ANN Architecture for Smart MIMO Channel estimation for AWGN and Flat Rayleigh is described for downlink LTE-Advanced System. Fig. 5, 6, 7, 8, 9, 10, 11 and 12 show the Throughput versus SNR results for comparison of different neural networks based channel estimation method with LS channel estimator and Ideal Channel for Closed Loop Spatial Multiplexing-Single User Multi-input Multi-output $(2 \times 2$ and $4 \times 4)($ CLSMSUMIMO) for AWGN and Flat Rayleigh Channel.

Fig. 5, 6, 7 and 8 show the Throughput versus SNR results for comparison of different neural networks based channel estimation method with LS channel estimator and Ideal Channel for Closed Loop Spatial Multiplexing-Single User Multi-input Multi-output $(2 \times 2$ and $4 \times 4$ ) (CLSM-SUMIMO) for AWGN Channel. Fig. 6 and 8 are column chart representation of Fig. 5 and 7, respectively.

From Fig. 5, 6, 7 and 8, Cascade-forward neural network is the worst ANN for this application. Feedforward neural network is giving better performance 
than Cascade-forward neural network. But, Layered Recurrent Neural Network (LRN) is the most effective to improve performance and shows better results for SNR range $0 \mathrm{~dB}$ to $30 \mathrm{~dB}$ when compared to ANN based Channel estimators using Feed-forward neural network and Cascade-forward neural network and traditional method LS estimator. It gives $0.7104 \mathrm{Mbps}$, 1.958 Mbps, 4.09 Mbps, 6.486 Mbps, 8.847 Mbps, 9.76 Mbps and $9.76 \mathrm{Mbps}$ for CLSM-MIMO $(2 \times 2)$ and 1.349 Mbps, 3.587 Mbps, 7.828 Mbps, 10.07 Mbps, 16.46 Mbps, 18.51 Mbps and 18.51 Mbps for CLSMMIMO $(4 \times 4)$ at $0,5,10,15,20,25$ and $30 \mathrm{~dB}$ respectively which are the best performances when compared to Feed-forward neural network, Cascadeforward neural network and traditional Least Square (LS) methods.

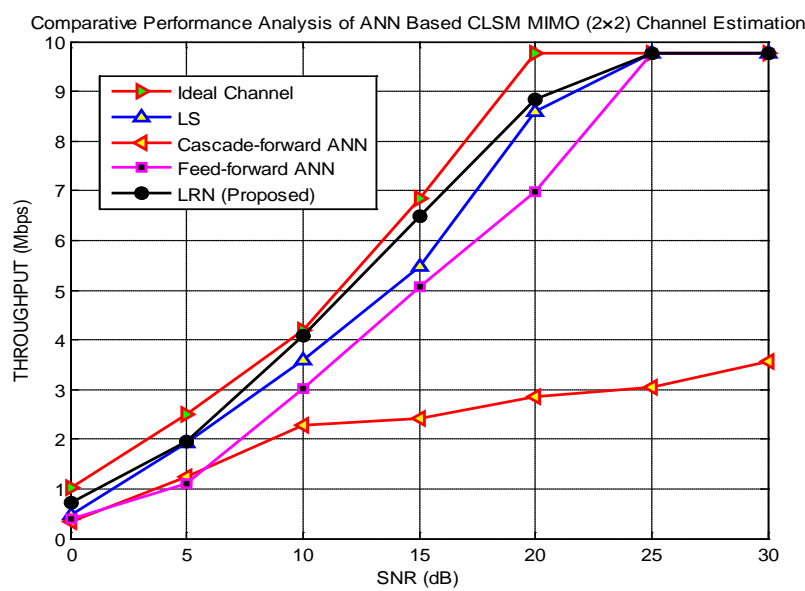

Figure 5. Throughput v/s SNR for ANN based channel estimators in comparison with LS and Perfect Channel in CLSM-MIMO $(2 \times 2)$ for AWGN Channel

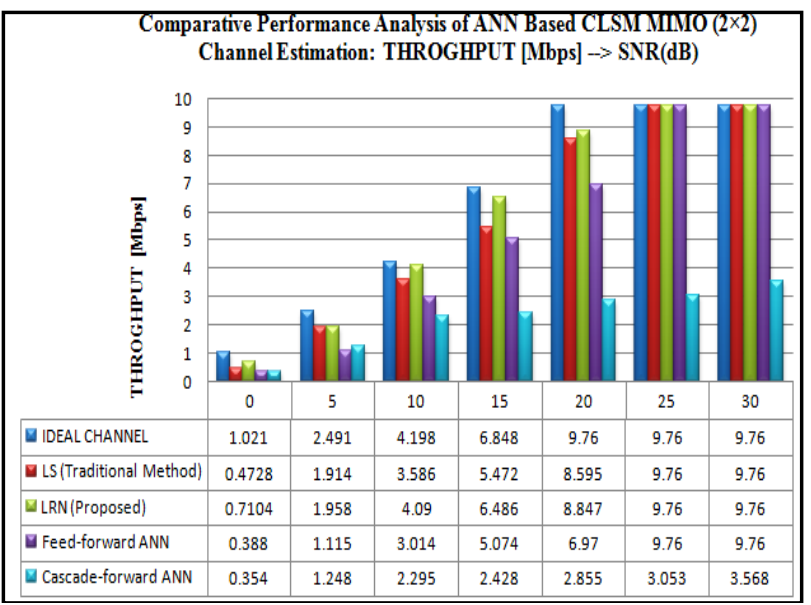

Figure 6. Throughput v/s SNR for ANN based channel estimators in comparison with LS and Perfect Channel in CLSM-MIMO $(2 \times 2)$ for AWGN Channel

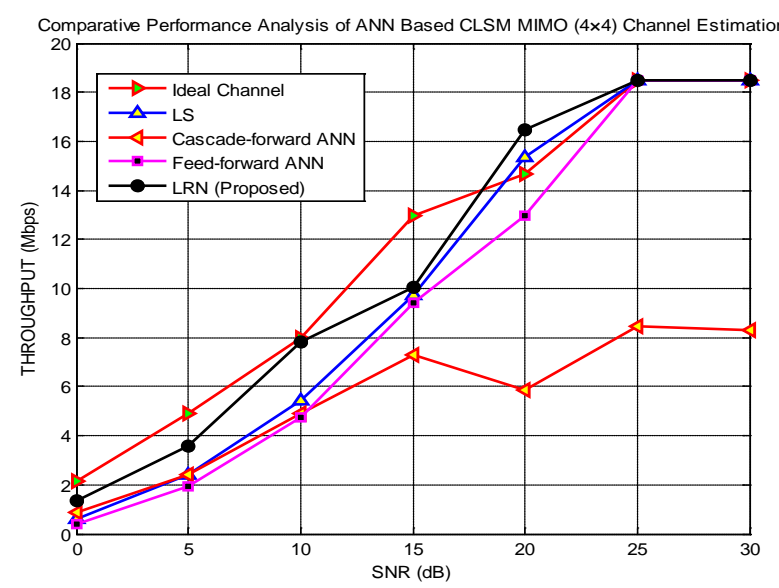

Figure 7. Throughput v/s SNR for ANN based channel estimators in comparison with LS and Perfect Channel in CLSM-MIMO $(4 \times 4)$ for AWGN Channel

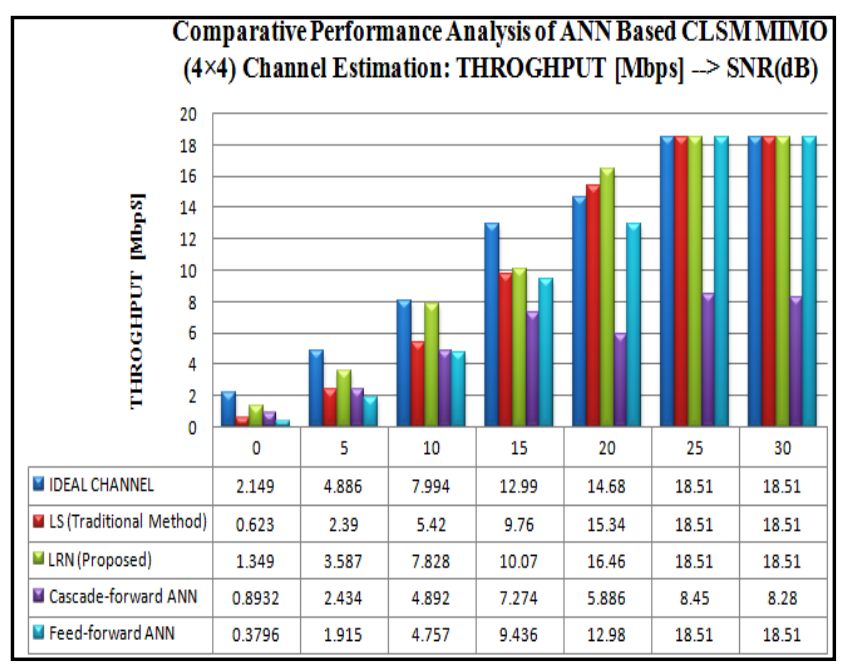

Figure 8. Throughput v/s SNR for ANN based channel estimators in comparison with LS and Perfect Channel in CLSM-MIMO $(4 \times 4)$ for AWGN Channel

Fig. 9, 10, 11 and 12 show the Throughput versus SNR results for comparison of different neural networks based channel estimation method with LS channel estimator and Ideal Channel for Closed Loop Spatial Multiplexing-Single User Multi-input Multi-output $(2 \times 2$ and $4 \times 4)($ CLSM-SUMIMO) for Flat Rayleigh Channel. Fig. 10 and 12 are column chart representation of Fig. 9 and 11 , respectively.

From Fig. 9, 10, 11 and 12, Cascade-forward neural network is the worst ANN for this application. Feedforward neural network is giving better performance than Cascade-forward neural network. But, Layered Recurrent Neural Network (LRN) is the most effective to improve performance and shows better results for SNR range $0 \mathrm{~dB}$ to $30 \mathrm{~dB}$ when compared to ANN based Channel estimators using Feed-forward neural network and Cascade-forward neural network and traditional method LS estimator. It gives $1.062 \mathrm{Mbps}$, 2.054 Mbps, 3.192 Mbps, 4.87 Mbps, 6.57 Mbps, 8.435 Mbps and 9.578 Mbps for CLSM-MIMO $(2 \times 2)$ and 1.562 Mbps, 2.637 Mbps, 5.182 Mbps, 7.63 Mbps, 11.14 Mbps, 14.29 Mbps and 16.89 Mbps for CLSM- 
MIMO (4×4) at $0,5,10,15,20,25$ and $30 \mathrm{~dB}$ respectively which are the best performances when compared to Feed-forward neural network, Cascadeforward neural network and traditional Least Square (LS) methods. But after SNR $30 \mathrm{~dB}$, LRN is not much effective as in 0 to $30 \mathrm{~dB}$ SNR. From Fig. 9, It is also giving throughput performance better than Ideal Channel for SNR range from $25 \mathrm{~dB}$ to $40 \mathrm{~dB}$ for CLSM-MIMO $(2 \times 2)$ in Flat Rayleigh channel.

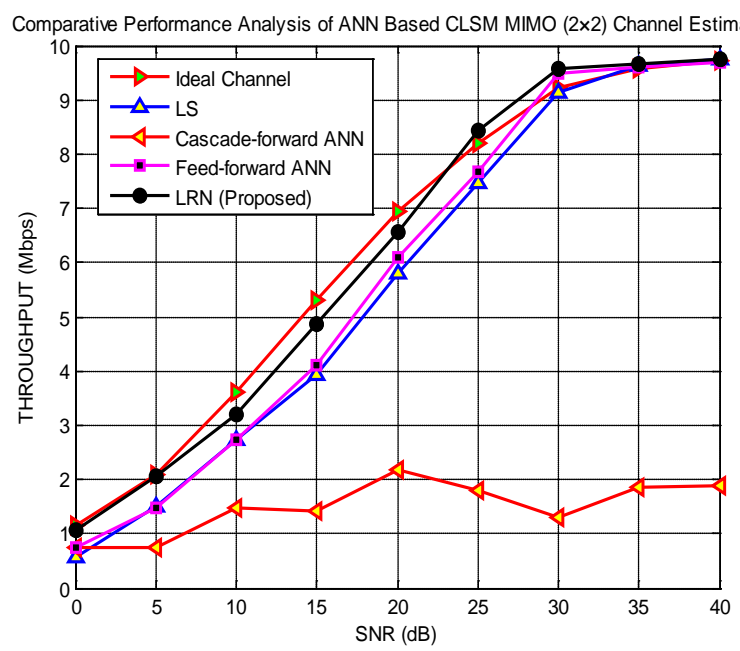

Figure 9. Throughput v/s SNR for ANN based channel estimators in comparison with LS and Perfect Channel in CLSM-MIMO $(2 \times 2)$ for Flat Rayleigh Channel

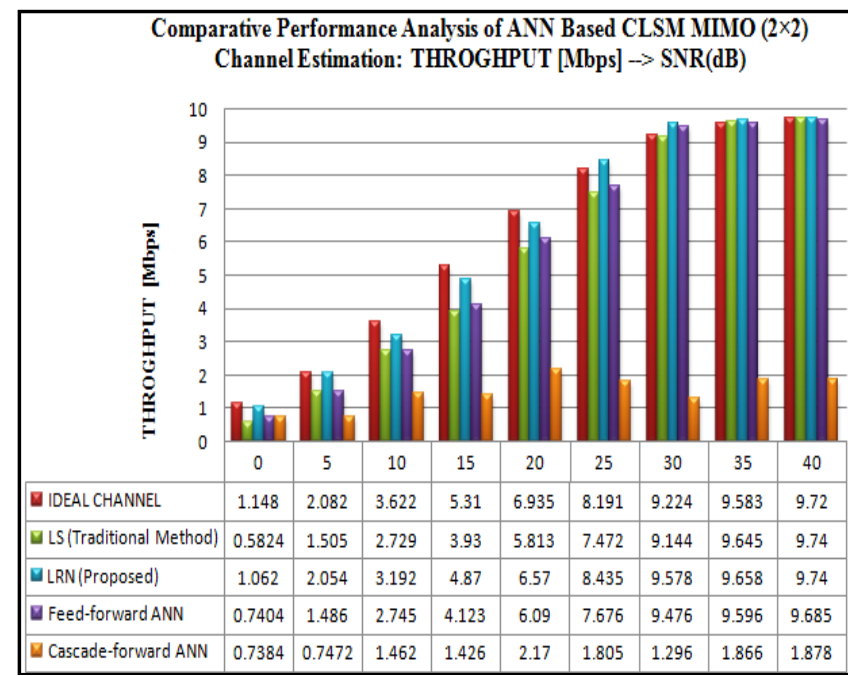

Figure 10. Throughput v/s SNR for ANN based channel estimators in comparison with LS and Perfect Channel in CLSM-MIMO $(2 \times 2)$ for Flat Rayleigh Channel

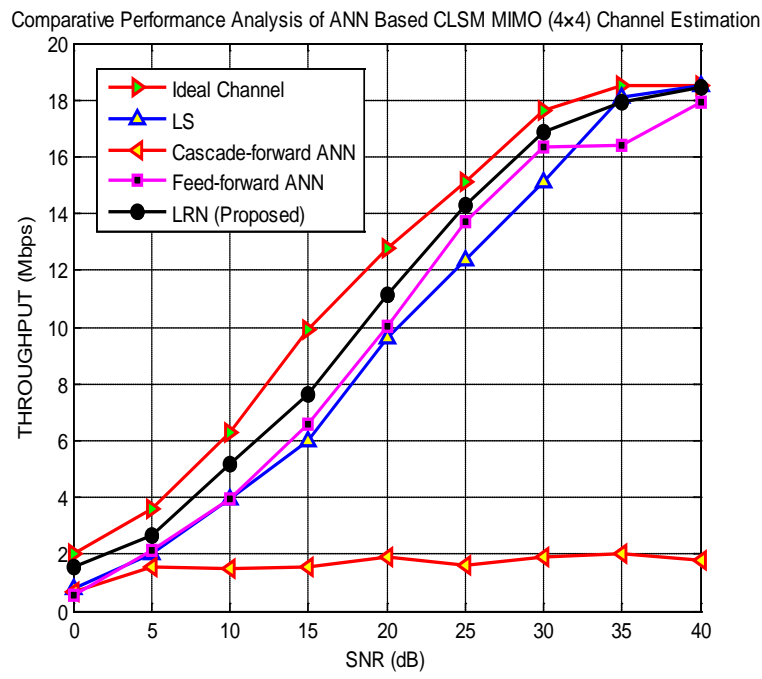

Figure 11. Throughput v/s SNR for ANN based channel estimators in comparison with LS and Perfect Channel in CLSM-MIMO (4×4) for Flat Rayleigh Channel

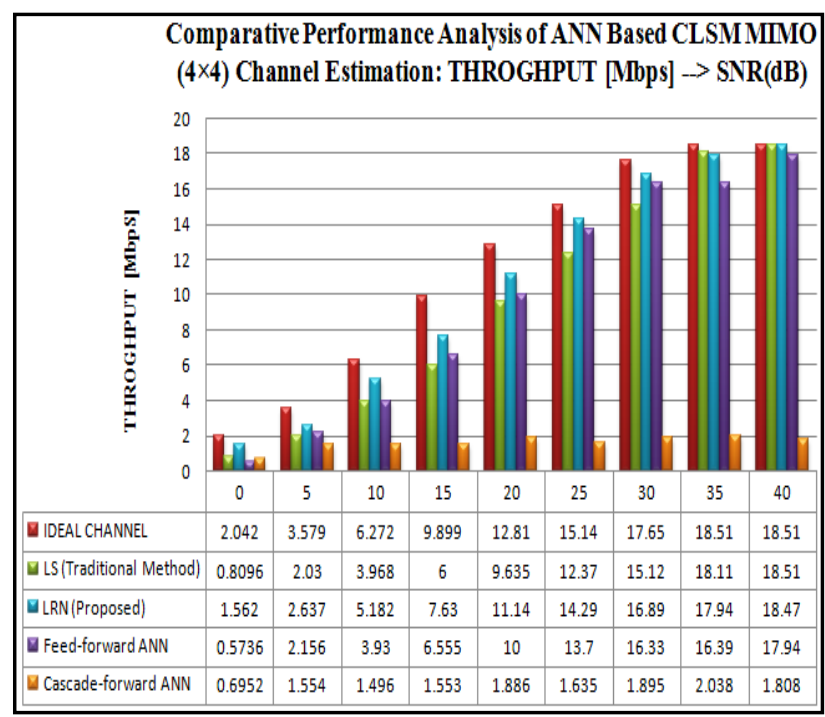

Figure 12. Throughput v/s SNR for ANN based channel estimators in comparison with LS and Perfect Channel in CLSM-MIMO $(4 \times 4)$ for Flat Rayleigh Channel

\section{CONCLUSION}

In this paper, Comparative Effect of ANN Architecture for Smart MIMO Channel Estimation AWGN and Flat Rayleigh in downlink LTE-Advanced System is introduced. This method is based on a learning process that uses a training sequence for adaptation to achieve a desired performance. Comparative analysis of proposed estimation method, Layered Recurrent Neural Network (LRN) with traditional LS channel estimation technique and other different two ANN Architecture based channel estimators are carried out for Closed Loop Spatial Multiplexing-Single User Multi-input Multi-output ( $2 \times 2$ and $4 \times 4)(C L S M-S U M I M O)$. Simulation results show better performance in terms of throughput for the proposed Layered Recurrent Neural Network (LRN). It 
shows better results when compared to traditional LS channel estimation technique and ANN based Channel estimation techniques using Feed-forward neural network and Cascade-forward neural network. Because, In the LRN, there is a feedback loop, with a single delay, around each layer of the network except for the last layer. The performance of channel estimator can be further enhanced by using Hybrid techniques for training Artificial Neural Networks.

\section{ACKNOWLEDGEMENT}

The authors are grateful to the staff of Department of Electrical Engineering, Faculty of Technology and Engineering, The Maharaja Sayajirao University of Baroda, for their support. This work has been supported by the grant from Department of Science and Technology (DST), Government of India, under the Women Scientist (WOS-A) Scheme.

\section{REFERENCES}

[1] 3GPP TR 36.913, “3GPP; Technical Specification Group Radio Access Network. Requirements for further advancements for Evolved Universal Terrestrial Radio Access (E-UTRA)," v.9.0.0, December 2009.

[2] M. Rumney, "LTE and the Evolution to 4G Wireless: Design and Measurement Challenges”, Agilent Technologies Publication, 2009.

[3] David Martin-Sacristan, Jose F. Monserrat, Jorge Cabrejas-Penuelas, Daniel Calabuig, Salvador Garrigas, and Narcis Cardona, "3GPP LTE and LTE-Advanced”, January 2009.

[4] Jeanette Wannstrom, "LTE-Advanced", May 2012 http://www.3gpp.org/LTE-Advanced

[5] 3GPP TS 36.211, Evolved Universal Terrestrial Radio Access (EUTRA), "Physical Channels and Modulation”, (Release 8).

[6] A. Omri, R. Bouallegue, R. Hamila and M. Hasna, "Estimation of highly selective channels for downlink LTE MIMO-OFDM system by a robust neural network", International Journal of Wireless \& Mobile Networks( IJWMN ), Vol.2, No. 1 (2011), pp. 31 - 38.

[7] Barnali Dey, Awanish Kumar, Prashant Kumar, Sanjit Lal, Naveen Kumar, Bikash Sharma, "Channel Estimation using LS and MMSE Algorithm”, International Symposium on Devices MEMS, Intelligent Systems \& Communication (ISDMISC) 2011.

[8] Albert Serra Pagès, “A Long Term Evolution Link Level Simulator”, Universitat Politècnica de Catalunya February, 2009.

[9] Asad Mehmood Waqas Aslam Cheema, "Channel Estimation in for LTE Downlink”, Blekinge Institute of Technology, September 2009.

[10] Johanna Ketonen, "Equalization and Channel estimation algorithms and implementations for
Cellular MIMO-OFDM downlink”, University of Oulu Graduate School; Faculty of Technology, Department Of Communications Engineering; Centre For Wireless Communications; Infotech Oulu, June 2012.

[11] Saqib Saleem, "Channel Estimation using Adaptive Filtering for LTE-Advanced”, International Journal of Computer Science (IJCSI) Issues, Vol. 8, Issue 3, No. 2, May 2011.

[12] A. Omri, R. Bouallegue, R. Hamila and M. Hasna, "Channel estimation for LTE uplink system by perceptron neural network”, International Journal of Wireless \& Mobile Networks (IJWMN), Vol.2, No. 3, pp. 155 - 165, August 2010. http.dx.doi.org/10.5121/ijwmn.2010.2311.

[13] Kanchan Sharma, Shweta Varshney, “Artificial Neural Network Channel Estimation for OFDM System”, International Journal of Electronics and Computer Science Engineering (IJECSE), ISSN 2277-1956/V1N3-1686-1691.

[14] J.SUN, Y.Dong-Feng, "Neural Network Channel Estimation Based on Least Mean Error Algorithm in the OFDM Systems”, International Symposium on Neural Networks (ISNN) 2006, Chengdu, China.

[15] Sun, J., \& Yuan, D. F. (2006). Neural network channel estimation based on least mean error algorithm in the OFDM systems. Advances in Neural Networks. Berlin: Springer.

[16] C. Mehlführer, M. Wrulich, J. C. Ikuno, D. Bosanska and M. Rupp, "Simulating the Long Term Evolution Physical Layer," in Proc. of the 17th European Signal Processing Conference (EUSIPCO 2009), Aug. 2009, Glasgow, Scotland.

[17]LTE World, The Seven Modes of MIMO in LTE, [online]. Available: http://lteworld.org/whitepaper/seven-modes-mimolte.

[18] J. Zyren, "Overview of the 3GPP Long Term Evolution Physical Layer”, Freescale Semiconductor, Inc.

[19] Satish K. Shah, Pooja S. Suratia, Nirmalkumar S. Reshamwala, "Comparative Performance Analysis of ANN Based MIMO Channel Estimation for downlink LTE- Advanced System employing Genetic Algorithm”, proceeding of International Conference On Soft Computing and Software Engineering [SCSE'13].

[20] Nirmalkumar S. Reshamwala, Pooja S. Suratia, Satish K. Shah, "Performance Analysis of ANN Based MIMO Channel Estimation for downlink LTE-Advanced System”, National Level Paper Contest called "Soft Computing for processing, Security Networking and Communication: (SCPSNC_2013), IETE Vadodara and Faculty of Technology, Electrical Engineering Department, M S University of Baroda, Vadodara, 20th January, 2013.

[21] Desi Pramudiwati, "LTE System Performance In Relation To Wideband Channel Properties”, Wireless and Mobile Communications Group, 
Department of Telecommunications, Faculty of Electrical Engineering, Mathematics and Computer Science, Delft University of Technology, 2011.

[22][Online].Available: http://www.nt.tuwien.ac.at/about-us/staff/josepcolom-ikuno/lte-simulators/

[23] C. Mehlfuhrer, J. Colom Ikuno, M. Simko, S. Schwarz, M. Wrulich, M. Rupp, “The Vienna LTE Simulators - Enabling Reproducibility in Wireless Communications Research”, EURASIP Journal on Advances in Signal Processing, Vol. 2011, pages 1 - 13, 2011.

[24]3GPP Document TS 36.213 “Evolved Universal Terrestrial Radio Access (EUTRA); Physical Layer Procedure (release 8).”

[25] Farooq Khan, "LTE for 4G Mobile Broadband Air Interface Technologies and Performance”, Telecom R\&D Center Samsung Telecommunications, America, Cambridge University Press 2009.

[26]3GPP TS 36.101 V8.4.0 (2008-12), "Evolved Universal Terrestrial Radio Access (E-UTRA); User Equipment (UE) radio transmission and reception".

[27]3GPP TS 36.104 V8.4.0 (2008-12), “Evolved Universal Terrestrial Radio Access (E-UTRA); Base Station (BS) radio transmission and reception".

[28] Hoo-Jin Lee, Shailesh Patil, and Raghu G. Raj, "Fundamental overview and simulation of MIMO systems for Space-Time coding and Spatial Multiplexing", Wireless Networking and Communications Group (WNCG), Dept. of Electrical and Computer Engineering,The University of Texas at Austin, 2003.

[29] L.Hanzo, M.Münster, B.J.Choi and T. keller, "Chapter 17: Uplink Detection Techniques for Multi-User SDMA-OFDM”, “OFDM and MCCDMA for Broadband Multi-user Communications, WLANs and Broadcasting”, John Wiley and Sons, Ltd. 2003.

[30] Albert van Zelst, "MIMO OFDM for Wireless LANs”, PROEFSCHRIFT, April 2004.

[31] Na wei, "MIMO Techniques for UTRA Long Term Evolution”, Dissertation, September 2007.

[32] 3GPP R4-070141, "Radio Propagation Modeling for E-UTRA performance requirement definition”, February 2007.

[33] P. Gupta, N. Sinha, An improved approach for nonlinear system identification using neural networks in Journal of the Franklin Institute 336(1999) 721-734.

[34] Dave Anderson and George McNeill, “Artificial Neural Networks Technology”, Kaman Sciences Corporation 258 Genesse Street Utica, New York 13502-462.

[35] David J. Montana, Lawrence Davis, “Training Feedforward Neural Networks Using Genetic Algorithms”, BBN Systems and Technologies Corp, 10 Mouiton St. Cambridge, MA 02138.
[36] Jatinder N.D. Guptaa, Randall S. Sextonb, "Comparing backpropagation with a genetic algorithm for neural network training”, International Journal of Management Science, Omega 27 (1999) 679-684.

[37] O. De Jesus and M. T. Hagan, "Backpropagation Algorithms for a Broad Class of Dynamic Networks," IEEE Transactions on Neural Networks, vol.18, no.1, pp.14 -27, Jan.2007.

[38] MathWorks, Neural Network Toolbox 7.0, MathWorks Announces Release 2010a of the MATLAB and Simulink Product Families, 2010, MathWorks, Inc. www.mathworks.com/trademarks.

[39] Omaima N. Ahmad AL-Allaf, "Cascade-Forward vs. Function Fitting Neural Network for Improving Image Quality and Learning Time in Image Compression System", Proceedings of the World Congress on Engineering 2012 Vol II, WCE 2012, July 4 - 6, 2012, London, U.K.

[40] D. NAGESH KUMAR, K. SRINIVASA RAJU, T. SATHISH, "River Flow Forecasting using Recurrent Neural Networks", Water Resources Management 18: 143-161, 2004.

[41] Mikael Bod'en, “A guide to recurrent neural networks and backpropagation", School of Information Science, Computer and Electrical Engineering Halmstad University,November 13, 2001.

[42] C. Lim, D.Han,’Robust LS channel estimation with phase rotation for single frequency network in OFDM ", IEEE Transactions on Consumer Electronics, Vol. 52 , pp. 1173 - 1178, 2006. http.dx.doi.org/10.1109/TCE.2006.273130.

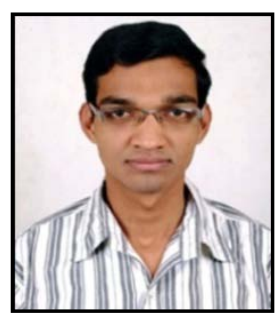

Authors' Introduction Nirmalkumar S. Reshamwala was born in Surat, Gujarat, India, on May $10^{\text {th }}$, 1990. He has obtained B.E. (Electronics \& Communication) from Dharmsinh Desai University, Nadiad, Gujarat, India in May-2011 and has completed M.E. (Electrical Engineering-Automatic Control \& Robotics) in July-2013. Currently, he is a Assistant Professor in Electronics \& Communication department at Sarvajanik College of Engineering \& Technology, Surat, Gujarat, India. His areas of interests are Wireless Communication (LTE, 4G, etc), Networking, Channel Estimation and Equalization and Soft-Computing.

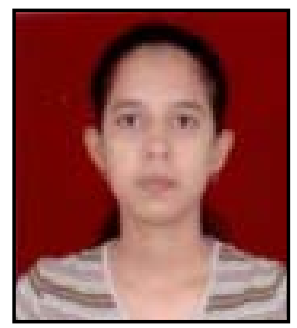

Pooja S. Suratia is currently a Research Scholar in Department of Electrical Engineering, Faculty of Technology and Engineering, The Maharaja Sayajirao University of Baroda, Vadodara, Gujarat, India. She received 
Masters of Engineering Degree in Automatic Control and Robotics from The Maharaja Sayajirao University of Baroda (2009), Gujarat, India and a Bachelor of Engineering in Electronics and Communication from Sa'd Vidya Mandal Institute of Technology, Bharuch, Gujarat (2006), India. Her current research interests focus on MIMO Wireless Communication and Optimization of future wireless systems.

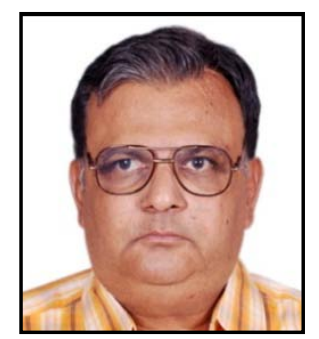

Prof. Satish Shah is a professor in the Electrical Engineering Department at Faculty of Technology, MS University of Baroda for last Twenty Five years. He is a fellow of IE(I) for past Fourteen Years and has also served as the member of Committee of Vadodara local center for more than Ten years in past. He has guided more than hundred projects at UG/PG level and completed a research project on DSP based Active Power filter sponsored by AICTE, New Delhi. He has written three books on Embedded System design / Microprocessors / Microcontrollers and presented/published more than 35 research papers in national / international conferences / Journals. He has attended and organized several seminars, workshops, and symposiums for UGC, AICTE, IETE, and MSU. He is a fellow of other technical associations such as: IETE, ISA and IEEE (NY) \& ISTE. He has served as the member, Hon Secretary and Treasurer of their local executive committees for a span of ten years. 